USTC-ICTS-08-17

\title{
On the low energy brane/anti-brane dynamics
}

\author{
J. X. Lu1, Bo Ning 2 and Guan-Nan Zhong 3 \\ Interdisciplinary Center for Theoretical Study \\ University of Science and Technology of China, Hefei, Anhui 230026, China
}

\begin{abstract}
We study the dynamical behavior of a pair of Dp-brane and anti Dp-brane $(0 \leq p \leq 6)$ moving parallel to each other in the region where the brane and anti-brane annihilation will not occur and the low energy description is valid. Given this, we perform a general analysis, in the center of mass frame, of the behavior of the effective potential with respect to the relative brane separation and find that the classical orbits of this system are in general unbound except for $p=6$ case for which classical bound orbits exist. The non-linearity of the low energy DBI action for D-brane is important for the underlying dynamics. We solve also the explicit orbits for $p=6$ case.
\end{abstract}

\footnotetext{
${ }^{1}$ E-mail: jxlu@ustc.edu.cn

${ }^{2}$ E-mail: nwaves@mail.ustc.edu.cn

${ }^{3}$ E-mail: zhonggn@email.jlu.edu.cn
} 


\section{Introduction}

It is known that two parallel D-branes separated by a distance feels no force between them, independent of their separation. This is due to the BPS nature or the preservation of certain number of space-time supersymmetries of this system, and goes by the name "no-force" condition. This was shown initially for brane supergravity configurations through a probe [1, 2] and later through the full string level computations as an open string one-loop annulus diagram with one end of the string located at one D-brane and the other end at the other D-brane making use of the "usual abstruse identity" [3]. With this, one can easily infer that when one of branes in the above is replaced by the corresponding anti-brane, there must be a separation-dependent non-vanishing force to arise since such a system breaks all the space-time supersymmetry. The corresponding forces can easily be computed given our knowledge of computing forces between two identical branes.

Analyzing the force behavior on the brane separation indicates the well-known fact that the brane and anti-brane will start to annihilate each other when the separation is on the order of string scale [4, 5]. Therefore to prevent this from happening, we should limit the brane separation to be much larger than the string length $l_{s}=\sqrt{\alpha^{\prime}}$ with the string parameter $\alpha^{\prime}$ related to the tension $T$ by $\alpha^{\prime}=1 /(2 \pi T)$. When this is met, the attractive force between the brane and the anti-brane can well be approximated by its long-distance form and the brane or anti brane itself is believed to be described by its low energy Dirac-Born-Infeld (DBI) action. So we have a well-defined system of D-brane and anti D-brane plus their interaction in the region where the brane and anti-brane annihilation will not occur and the low energy description is valid.

The DBI action, describing the low energy behavior of D-brane, is nonlinear and it is interesting to explore how D-brane behaves under this action. For this purpose, we analyze the dynamical behavior of a pair of Dp-brane and anti Dp-brane $(0 \leq p \leq 6)$ placed parallel to each other under the conditions mentioned above 4 . In section 2 , we set up the system so we can perform an analysis in the later sections. In section 3, we give a general analysis based on the effective potential and find that the classical orbits are in general unbound except for $p=6$ case where classical bound orbits exist. We also derive the conditions for which the respective orbits can exist. In section 4 , we solve the explicit orbits for the $p=6$ case as an example and find that they are consistent with our general analysis given in the previous section. We conclude this paper in section 5 .

\footnotetext{
${ }^{4} \mathrm{~A}$ probe approach with similar considerations has been given in [6, 7] where the orbits of a probe anti $p^{\prime}$-brane in a background of a stack of $N$ Dp branes with either $p^{\prime}=p$ or $p^{\prime} \neq p$ and $g_{s} N \gg 1$ have been considered, respectively. Our focus here is on the classical orbits of a pair of Dp-brane and anti Dp-brane in a different region of $g_{s} N \ll 1$ with $N=1$ and a flat background. Even so, many conclusions drawn here such as the $p=6$ case is singled out for the existence of bound orbits are the same as theirs.
} 


\section{Preliminaries}

We consider a system consisting of a pair of Dp-brane and anti Dp-brane $(0 \leq p \leq 6)$ moving parallel to each other in the region where the brane and anti-brane annihilation will not occur and the low energy description for the branes is valid. Given this, even though the brane tension is now large due to the weak string coupling limit, the spacetime around each brane still remains as flat so long we don't probe a distance much smaller than the string scale [5]. Our interest is in the classical relative motion of the two branes under their mutual attractive long-distance interaction and so we don't expect to excite the gauge modes on the brane. So the DBI action for either of the branes is reduced to

$$
\mathrm{S}_{\mathrm{DBI}}=-T_{p} \int d^{p+1} \sigma \sqrt{-\operatorname{det} \gamma_{\mu \nu}}
$$

where the induced metric on the brane is $\gamma_{\mu \nu}=\partial_{\mu} X^{M} \partial_{\nu} X^{N} \eta_{M N}$ with $\eta_{M N}$ the spacetime Minkowski flat metric and the brane tension $T_{p}=1 /\left[g_{s}(2 \pi)^{p} \alpha^{\prime \frac{p+1}{2}}\right]$ with $g_{s}$ the string coupling. We take now the so-called static gauge, i.e. $\sigma^{\mu}=X^{\mu}$ with $\mu=0,1, \cdots p$ and the underlying dynamics under consideration says that the coordinates transverse to the brane depend only on time, i.e., $X^{i}=X^{i}(\tau)$ with $\tau=\sigma^{0}=t$ and $i=p+1, \ldots, 9$. If we denote $\dot{X}^{i}=d X^{i} / d \tau$, we have from the above the following

$$
S_{\mathrm{DBI}}=-T_{p} V_{p} \int d \tau \sqrt{1-\dot{X}^{i} \dot{X}^{i}}
$$

where the brane spatial volume $V_{p}=\int d^{p} \sigma$. In the following, for simplicity, we also use the vector notation $\dot{\vec{X}}$ in replace of its components $\dot{X}^{i}$.

At any moment $\tau$, we denote the Dp-brane and the anti Dp-brane locations in the direction transverse to the branes as $\vec{X}_{1}$ and $\vec{X}_{2}$, respectively. With this, the interaction potential between them at a separation $r=\left|\vec{X}_{2}-\vec{X}_{1}\right|$ can be obtained the way described in the Introduction and is given by

$$
V=-V_{p+1} \int_{0}^{\infty} \frac{d s}{s}(2 \pi s)^{-\frac{p+1}{2}} e^{-\frac{s}{4 \alpha^{\prime}}\left(\frac{r^{2}}{2 \pi^{2} \alpha^{\prime}}-1\right)} \prod_{n=1}^{\infty}\left(\frac{1-q^{2 n-1}}{1-q^{2 n}}\right)^{8},
$$

where the p-brane worldvolume $V_{p+1}=V_{p} \int d \tau, q=e^{-s / 4 \alpha^{\prime}}$ and the integration variable $s$ is the proper time in the open string channel. When the separation $r$ approaches the string scale, the interaction force will be divergent, indicating the occurring of the tachyon condensation [4, 5]. For the interest of this paper, we have $r \gg l_{s}=\sqrt{\alpha^{\prime}}$. So the main contribution to the integral in the above potential comes from small $s$. Note that [5],

$$
g(s)=\prod_{n=1}^{\infty}\left(\frac{1-q^{2 n-1}}{1-q^{2 n}}\right)^{8} \rightarrow\left(s / 2 \pi \alpha^{\prime}\right)^{4} \quad \text { as } \quad s \rightarrow 0,
$$


So the long-distance potential is attractive, as expected, and is given as $V(r)=-V_{p+1} \frac{\Omega}{r^{7-p}}$ with $\Omega=2^{2(3-p)} \pi^{\frac{5-3 p}{2}} \alpha^{\prime 3-p} \Gamma\left(\frac{7-p}{2}\right)$. Therefore the complete action for the Dp-D̄p system $(0 \leq p \leq 6)$ in the region of interest is:

$$
\mathrm{S}=-T_{p} V_{p} \int d \tau\left(\sqrt{1-\dot{\vec{X}}_{1} \cdot \dot{\vec{X}}_{1}}+\sqrt{1-\dot{\vec{X}}_{2} \cdot \dot{\vec{X}}_{2}}\right)+V_{p} \int d \tau \frac{\Omega}{\left|\vec{X}_{2}-\vec{X}_{1}\right|^{7-p}},
$$

which looks like a classical two-body mechanical problem with now the non-linear kinetic terms. To simplify the discussion, as usual for a two-body system, we choose the center of mass coordinate $\vec{R}$ and the relative coordinate $\vec{r}=2 \vec{\rho}$ as

$$
\begin{aligned}
\vec{R}=\frac{\vec{X}_{1}+\vec{X}_{2}}{2}, & \vec{X}_{1}=\vec{R}+\frac{\vec{r}}{2}=\vec{R}+\vec{\rho} \\
\vec{r}=2 \vec{\rho}=\vec{X}_{1}-\vec{X}_{2}, & \vec{X}_{2}=\vec{R}-\frac{\vec{r}}{2}=\vec{R}-\vec{\rho},
\end{aligned}
$$

where for later convenience, we have also introduced $\vec{\rho}$ which is half of the relative separation vector $\vec{r}$. Then the above action, denoting $\rho=|\vec{\rho}|$, becomes

$$
\begin{aligned}
\mathrm{S}= & -T_{p} V_{p} \int d \tau[\sqrt{1-(\dot{\vec{R}}+\dot{\vec{\rho}}) \cdot(\dot{\vec{R}}+\dot{\vec{\rho}})}+\sqrt{1-(\dot{\vec{R}}-\dot{\vec{\rho}}) \cdot(\dot{\vec{R}}-\dot{\vec{\rho}})}] \\
& +V_{p} \int d \tau \frac{\Omega}{(2 \rho)^{7-p}}
\end{aligned}
$$

Note that the generalized momentum corresponding to coordinate $\vec{R}$ is conserved:

$$
\vec{P}_{\vec{R}}=\frac{\partial \mathrm{L}}{\partial \dot{\vec{R}}}=V_{p} T_{p}\left(\frac{\dot{\vec{R}}+\dot{\vec{\rho}}}{\sqrt{1-(\dot{\vec{R}}+\dot{\vec{\rho}}) \cdot(\dot{\vec{R}}+\dot{\vec{\rho}})}}+\frac{\dot{\vec{R}}-\dot{\vec{\rho}}}{\sqrt{1-(\dot{\vec{R}}-\dot{\vec{\rho}}) \cdot(\dot{\vec{R}}-\dot{\vec{\rho}})}}\right) \equiv \vec{c},
$$

where $\vec{c}$ is a constant vector. The equation of motion with respect to $\vec{\rho}$ is

$$
T_{p} \frac{d}{d \tau}\left(\frac{\dot{\vec{R}}+\dot{\vec{\rho}}}{\sqrt{1-(\dot{\vec{R}}+\dot{\vec{\rho}}) \cdot(\dot{\vec{R}}+\dot{\vec{\rho}})}}-\frac{\dot{\vec{R}}-\dot{\vec{\rho}}}{\sqrt{1-(\dot{\vec{R}}-\dot{\vec{\rho}}) \cdot(\dot{\vec{R}}-\dot{\vec{\rho}})}}\right)+2 \frac{\Lambda}{\rho^{9-p}} \quad \vec{\rho}=0
$$

with $\Lambda=\frac{1}{2^{7-p}} \frac{7-p}{2} \Omega=2^{-(1+p)} \pi^{\frac{5-3 p}{2}} \alpha^{\prime 3-p} \Gamma\left(\frac{9-p}{2}\right)$.

In what follows, we focus on the case $\dot{\vec{R}}=0$ at a given initial time $\tau_{i}$, i.e. in the center of mass frame, implying $\vec{c}=0$ in (마). One can then show from the same equation $\dot{\vec{R}}=0$ at all time. This is entirely consistent with our usual picture that if we are in the center of mass frame at a given time, we are always so if only mutual interactions are present 5 .

\footnotetext{
${ }^{5}$ If we are not in the center of mass frame, the underlying dynamics seems rather complicated due to the non-linear DBI action and we try to explore this case elsewhere.
} 
For $\dot{\vec{R}}=0$, all the equations of motion reduce to the following one

$$
T_{p} \frac{d}{d t}\left(\frac{\dot{\vec{\rho}}}{\sqrt{1-\dot{\vec{\rho}}^{2}}}\right)+\frac{\Lambda}{\rho^{9-p}} \vec{\rho}=0 .
$$

This can be easily integrated out to give

$$
\frac{1}{\sqrt{1-\dot{\vec{\rho}}^{2}}}-\frac{\lambda}{\rho^{7-p}}=H
$$

where $H$ is an integral constant and $\lambda=\Lambda /\left[T_{p}(7-p)\right]=\frac{g_{s}}{4 \pi}\left(\pi \alpha^{\prime}\right)^{\frac{7-p}{2}} \Gamma\left(\frac{7-p}{2}\right) \sim g_{s} \alpha^{\prime \frac{7-p}{2}}$. The integral constant $H$ is actually the reduced Hamiltonian in the sense defined below: the Lagrangian from (7) when $\dot{\vec{R}}=0$ is

$$
\mathrm{L}=-2 T_{p} V_{p} \sqrt{1-\dot{\vec{\rho}}^{2}}+\frac{V_{p}}{2^{7-p}} \frac{\Omega}{\rho^{7-p}},
$$

and then we have the Hamiltonian

$$
\mathrm{H}=\vec{P}_{\vec{\rho}} \cdot \dot{\vec{\rho}}-\mathrm{L}=2 V_{p} T_{p}\left(\frac{1}{\sqrt{1-\dot{\vec{\rho}}^{2}}}-\frac{\lambda}{\rho^{7-p}}\right)=2 V_{p} T_{p} H
$$

There is an additional conserved quantity, the reduced angular momentum $J$, which can be obtained in the following way. Noticing the underlying dynamics to be planar in the sense $\dot{\vec{\rho}}^{2}=\dot{\rho}^{2}+\rho^{2} \dot{\theta}^{2}$, then the Lagrangian (12) can be re-expressed as

$$
\mathrm{L}=-2 T_{p} V_{p} \sqrt{1-\left(\dot{\rho}^{2}+\rho^{2} \dot{\theta}^{2}\right)}+\frac{V_{p}}{2^{7-p}} \frac{\Omega}{\rho^{7-p}},
$$

which implies the conserved angular momentum as

$$
\mathrm{J}=\frac{\partial \mathrm{L}}{\partial \dot{\theta}}=2 V_{p} T_{p} \frac{\rho^{2} \dot{\theta}}{\sqrt{1-\left(\dot{\rho}^{2}+\rho^{2} \dot{\theta}^{2}\right)}} \equiv 2 V_{p} T_{p} J,
$$

with the reduced conserved angular momentum as

$$
J=\frac{\rho^{2} \dot{\theta}}{\sqrt{1-\left(\dot{\rho}^{2}+\rho^{2} \dot{\theta}^{2}\right)}} .
$$

With this, we can further express the reduced Hamiltonian

$$
H=\frac{\sqrt{1+\frac{J^{2}}{\rho^{2}}}}{\sqrt{1-\dot{\rho}^{2}}}-\frac{\lambda}{\rho^{7-p}}
$$


and the corresponding effective potential is

$$
V_{\mathrm{eff}}=\sqrt{1+\frac{J^{2}}{\rho^{2}}}-\frac{\lambda}{\rho^{7-p}},
$$

which is our basis for analyzing the dynamical behavior of the system under consideration in the following section. To solve an orbit explicitly for allowed $H$ and $J$, we need the following equation

$$
\frac{d \rho}{d \theta}= \pm \frac{\rho^{2}}{J} \sqrt{\left(H+\frac{\lambda}{\rho^{7-p}}\right)^{2}-\left(1+\frac{J^{2}}{\rho^{2}}\right)}
$$

where the \pm correlate to the signs of $\dot{\rho}$ if we assume $J>0$. The explicit analytical orbits can be found only for $p=6$ case and they will be given in section 4 . As we will see, their behaviors are completely consistent with those obtained in section 3 based on the effective potential (18).

\section{The general dynamical behavior}

Given the preparation of the previous section, we are now ready to analyze the dynamical behavior of the system under consideration in the region of interest mentioned earlier. Our starting point is the effective potential (18),

$$
V_{\text {eff }}(\rho)=\sqrt{1+\frac{J^{2}}{\rho^{2}}}-\frac{\lambda}{\rho^{7-p}} .
$$

To avoid the brane annihilation from happening, we need to have $\rho \gg l_{s}=\sqrt{\alpha^{\prime}}=\left(a_{p} \lambda\right)^{1 /(7-p)}$ where we have used the expression for $\lambda$ given earlier and $a_{p}=4 /\left[g_{s} \pi^{\frac{5-p}{2}} \Gamma\left(\frac{7-p}{2}\right)\right] \gg 1$ with $g_{s} \ll 1$ the weak string coupling limit. So the second term in the effective potential appears to be small but as we will see it still has important dynamical effect in the region of interest.

To have some idea on the effective potential behavior 6 , let us first ignore the constraint on the allowed range for $\rho$. It is easy to examine that $V_{\text {eff }} \rightarrow 1$ as $\rho \rightarrow \infty$ for all $p$ under consideration while $V_{\text {eff }} \rightarrow-\infty$ as $\rho \rightarrow 0$ for $p<6$. For $p=6, V_{\text {eff }} \rightarrow-\infty$ if $J<\lambda$ and $V_{\text {eff }} \rightarrow \infty$ for $J>\lambda$ as $\rho \rightarrow 0$. As will be seen, only $J>\lambda$ will be relevant to our interest for this case. So the above behavior of the potential seems to indicate that for $p<6$, its extremum if exists at all should be a maximum, therefore only unbound orbits exist in the region of interest, while for $p=6$, the extremum should be a local minimum, therefore both bound and unbound orbits exist, depending on the initial energy.

\footnotetext{
${ }^{6}$ The non-linearity has an important consequence in the case of $p=5$ for which the effective potential develops a maximum when $J^{2}>2 \lambda$ while there is no extremum if it is ignored. For the rest of cases, this effect doesn't change the characteristic behavior of the potential whether it is considered or not.
} 
Let us now take a close look at this. The extremum of the potential can be determined from the vanishing of its first derivative with respective to $\rho$, i.e.,

$$
V_{\mathrm{eff}}^{\prime} \equiv \frac{d V_{\mathrm{eff}}}{d \rho}=-\frac{J^{2}}{\rho^{3}}\left[\frac{1}{\sqrt{1+\frac{J^{2}}{\rho^{2}}}}-\frac{7-p}{J^{2}} \frac{\lambda}{\rho^{5-p}}\right]=0 .
$$

The trivial extremum occurs at $\rho=\infty$ where the second derivative of the potential vanishes, and our interest is at the finite one satisfying

$$
\frac{J^{2}}{\sqrt{1+\frac{J^{2}}{\rho^{2}}}}=(7-p) \frac{\lambda}{\rho^{5-p}} .
$$

Assume that the extremum satisfying the above equation occurs at $\rho=\rho_{0}$, let us determine whether the extremum is a local maximum or a local minimum through the sign of the following second derivative of the potential at $\rho=\rho_{0}$ :

$$
\left.V_{\mathrm{eff}}^{\prime \prime} \equiv \frac{d^{2} V_{\mathrm{eff}}}{d \rho^{2}}\right|_{\rho=\rho_{0}}=-\frac{J^{2}}{\rho_{0}^{3}}\left[\frac{\frac{J^{2}}{\rho_{0}^{3}}}{\left(1+\frac{J^{2}}{\rho_{0}^{2}}\right)^{3 / 2}}+\frac{(7-p)(5-p)}{J^{2}} \frac{\lambda}{\rho_{0}^{6-p}}\right] .
$$

It is obvious that for $p<6$, the second derivative is always less than zero at $\rho_{0}$, therefore the extremum is a maximum. For $p=6$, the extremum exists at a non-zero finite $\rho_{0}$ only if $J>\lambda$ and we can now solve (22) to give

$$
\rho_{0}=J \sqrt{\left(\frac{J}{\lambda}\right)^{2}-1}
$$

With this, it is easy to check that the second derivative is actually positive, therefore giving the extremum a minimum. So our analysis here is completely consistent with our expectation from the asymptotical behavior of the potential given above. The characteristic behaviors of the potential for $p<6$ and $p=6$ are given in Fig. 1, respectively.

Given the above behavior of the potential, we come to analyze the dynamics of the brane/antibrane system under consideration in the region of interest case by case. Let us begin with $p=6$.

i) For $p=6$, the extremum occurs at $\rho_{0}$ as given in (24) when $J>\lambda$ and the extremum is a minimum. The properties of bound and unbound orbits are shown in Fig. 2.

In order to have a bound state, the reduced Hamiltonian should satisfy $V_{\text {eff }}\left(\rho_{0}\right) \leq H<1$. To avoid the brane annihilation, we need the minimal separation (denoted as $\rho_{\min }^{b}$ ) to satisfy $\rho_{\text {min }}^{b} \gg l_{s}=a_{6} \lambda$ with $a_{6}=4 / g_{s} \gg 1$. This gives $\rho_{0} \geq \rho_{\min }^{b} \gg l_{s}=a_{6} \lambda$ which from (24) implies

$$
J \gg \lambda \sqrt{a_{6}+\frac{1}{2}} \approx \lambda \sqrt{a_{6}}\left(1+\frac{1}{4 a_{6}}\right) .
$$

\footnotetext{
${ }^{7}$ We actually need $J \gg \lambda$ to avoid the annihilation. For $J \leq \lambda$, the potential has no extremum at a finite $\rho_{0}$ and falls either to zero or to $-\infty$ at small $\rho$, therefore the brane and anti-brane will inevitably annihilate each other.
} 

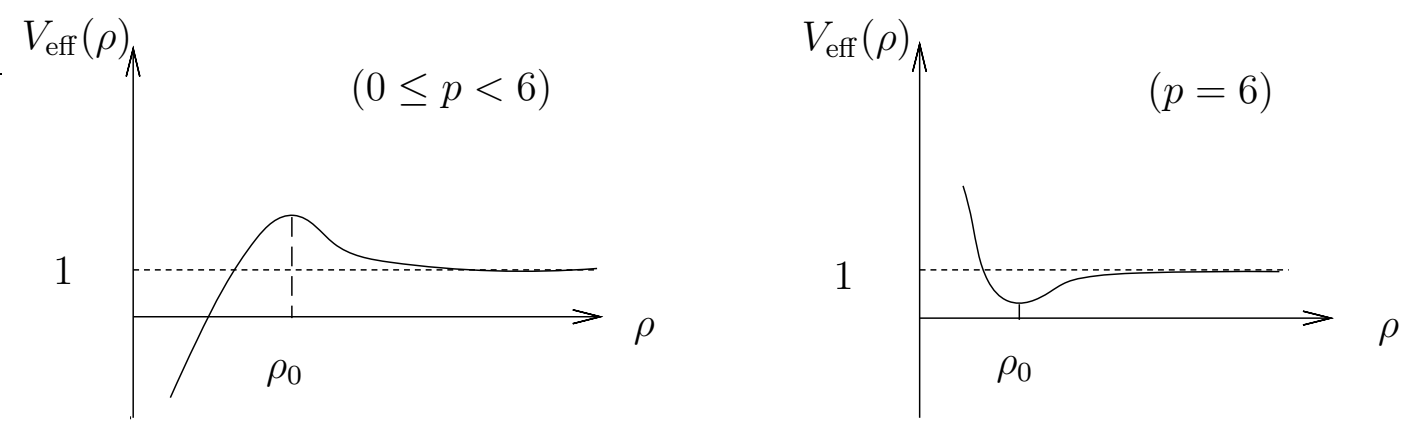

Figure 1: The respective characteristic behavior of the potential for $0 \leq p<6$ (left) and for $p=6$ when $J>\lambda$ (right).

Note that for a bound state $\left(\dot{\rho}_{\min }^{b}=0\right)$

$$
H=V_{\mathrm{eff}}\left(\rho_{\min }^{b}\right)=\sqrt{1+\left(\frac{J}{\rho_{\min }^{b}}\right)^{2}}-\frac{\lambda}{\rho_{\min }^{b}}<1,
$$

which implies $\rho_{\min }^{b}>\frac{J^{2}-\lambda^{2}}{2 \lambda}$. So we should have now $\rho_{\min }^{b}>\frac{J^{2}-\lambda^{2}}{2 \lambda} \gg a_{6} \lambda$. This gives

$$
J \gg \lambda \sqrt{2\left(a_{6}+\frac{1}{2}\right)} \approx \lambda \sqrt{2 a_{6}}\left(1+\frac{1}{4 a_{6}}\right),
$$

which is a bit stronger than the condition (25) as anticipated. Using (24), we have the minimal value of the effective potential as

$$
V_{\mathrm{eff}}\left(\rho_{0}\right)=\sqrt{1-\left(\frac{\lambda}{J}\right)^{2}} \approx 1-\frac{1}{2}\left(\frac{\lambda}{J}\right)^{2},
$$

which is very close to unit. So this gives the allowed range for the reduced Hamiltonian for bound orbits as

$$
\sqrt{1-\left(\frac{\lambda}{J}\right)^{2}} \leq H<1
$$

So (27) and (29) are the conditions for having bound orbits and avoiding the brane annihilation. We now come to the unbound orbits. Obviously we need to have $H \geq 1$ and this gives

$$
H=V_{\mathrm{eff}}\left(\rho_{\min }^{a}\right)=\sqrt{1+\left(\frac{J}{\rho_{\min }^{a}}\right)^{2}}-\frac{\lambda}{\rho_{\min }^{a}} \geq 1,
$$





Figure 2: The properties of unbound orbits for $p=5$ when $J^{2}>2 \lambda$ (left) and those of bound and unbound orbits for $p=6$ when $J \gg \lambda$ (right). For $p=6, \rho_{\min }^{b}$ is the minimal separation for the bound orbit and $\rho_{\min }^{a}$ is the correspondence for the unbound orbit.

where $\dot{\rho}_{\text {min }}^{a}=0$ with $\rho_{\text {min }}^{a}$ the present minimal brane separation. The above in turn implies

$$
\rho_{\min }^{a}=\frac{J^{2}-\lambda^{2}}{\lambda H+\sqrt{J^{2}\left(H^{2}-1\right)+\lambda^{2}}} \leq \frac{J^{2}-\lambda^{2}}{2 \lambda} .
$$

To avoid the brane annihilation, we need also $\rho_{\min }^{a} \gg l_{s}=a_{6} \lambda$. This, along with (31), first implies once again (27) between $J$ and $\lambda$ and then gives a constraint on $H$, when combined with $H \geq 1$, as

$$
1 \leq H \ll \sqrt{1+\frac{J^{2}}{\lambda^{2} a_{6}^{2}}}-\frac{1}{a_{6}} .
$$

So the conditions for having unbound orbits and for avoiding the brane annihilation are (27) and (32) for the present case.

ii) For $p=5$, the potential has a possible maximum. Eq.(21) implies that the maximum exists if $J^{2}>2 \lambda$ and if so it occurs at

$$
\rho_{0}=\frac{\frac{2 \lambda}{J}}{\sqrt{1-\left(\frac{2 \lambda}{J^{2}}\right)^{2}}} .
$$

The orbits are all unbound and to avoid the brane annihilation, we need to have $\left(a_{5} \lambda\right)^{1 / 2} \ll$ $\rho_{0}<\rho_{\min }$ with $a_{5}=4 / g_{s} \gg 1$. This gives rise to, using the expression for $\rho_{0}$ from (33),

$$
\frac{2}{a_{5}} \gg \frac{J^{2}}{2 \lambda}\left[1-\left(\frac{2 \lambda}{J^{2}}\right)^{2}\right] \text {. }
$$

Given $J^{2}>2 \lambda$ and $a_{5} \gg 1$, the above implies that $J^{2} /(2 \lambda)$ is very close to unit and if we set

$$
\frac{J^{2}}{2 \lambda}=1+\epsilon,
$$


then the very small $\epsilon$ satisfies, to leading order,

$$
0<\epsilon \ll \frac{1}{a_{5}}
$$

In addition, we need to have

$$
V_{\text {eff }}\left(\rho_{0}\right)>H \geq 1
$$

with now

$$
V_{\mathrm{eff}}\left(\rho_{0}\right)=\frac{1}{2}\left(\frac{J^{2}}{2 \lambda}+\frac{2 \lambda}{J^{2}}\right) .
$$

So the conditions for having unbound orbits and no occurrence of annihilation for the present case are (35), (36) and (37). The characteristic features of unbound orbits are given in Fig. 2.

iii) For $p=4$, the potential has a maximum. We have from (22) the maximum occurring at

$$
\rho_{0}^{2}=\frac{2 J^{2}}{\sqrt{1+4\left(\frac{J^{3}}{3 \lambda}\right)^{2}}-1} .
$$

The characteristic features of the potential and the unbound orbits are similar to those for $p=5$ as shown in Fig. 2. We have now

$$
V_{\text {eff }}\left(\rho_{0}\right)=\frac{2^{1 / 2}\left[1+\sqrt{1+4\left(\frac{J^{3}}{3 \lambda}\right)^{2}}+\frac{4}{3}\left(\frac{J^{3}}{3 \lambda}\right)^{2}\right]}{\left(1+\sqrt{1+4\left(\frac{J^{3}}{3 \lambda}\right)^{2}}\right)^{3 / 2}} .
$$

To have the unbound orbits and to avoid the brane annihilation, we need both

$$
V_{\text {eff }}\left(\rho_{0}\right)>H \geq 1
$$

with $V_{\text {eff }}\left(\rho_{0}\right)$ as given above, and the minimal brane separation satisfying $\rho_{\min }>\rho_{0} \gg l_{s}=$ $\left(a_{4} \lambda\right)^{1 / 3}$ with $a_{4}=8 /\left(g_{s} \pi\right) \gg 1$. This last constraint, combining with the expression for $\rho_{0}$ given in (39), gives

$$
6 \sqrt{2} \frac{J^{3}}{3 \lambda} \gg a_{4}\left[\sqrt{1+4\left(\frac{J^{3}}{3 \lambda}\right)^{2}}-1\right]^{3 / 2},
$$

which implies $J^{3} /(3 \lambda) \ll 1$. To leading order, we have

$$
\frac{J^{3}}{\lambda} \ll 3 \sqrt{\frac{3}{a_{4}}} \ll 1,
$$

and the maximum of the potential from (40) can now be approximated as $V_{\text {eff }}\left(\rho_{0}\right) \approx 1+\frac{1}{6}\left(\frac{J^{3}}{3 \lambda}\right)^{2}$. So the conditions in this case for having unbound orbits and for avoiding the brane annihilation are (41) and (43). 
iv) For $0 \leq p \leq 3$, the situation is similar to $p=4$ case and to the $\left(J^{2}>2 \lambda\right) p=5$ case. In other words, the potential has a maximum. The characteristic features of the potential and the unbound orbits are similar to those given in Fig. 2. However, the $\rho_{0}$ for which the potential takes maximum cannot for now be solved exactly. As will be seen, in the region of interest, it can still be solved approximately. As usual, to have unbound orbits and to avoid the brane annihilation, we need both $V_{\text {eff }}\left(\rho_{0}\right)>H \geq 1$ and $\rho_{\min }>\rho_{0} \gg l_{s}=\left(a_{p} \lambda\right)^{1 /(7-p)}$ with $a_{p}$ given earlier. Here $\rho_{\min }$ is once again the minimal brane separation for a given unbound orbit at which $\dot{\rho}_{\text {min }}=0$ and $H=V_{\text {eff }}\left(\rho_{\text {min }}\right)$. The last constraint can be re-expressed as

$$
\frac{\lambda}{\rho_{0}^{7-p}} \ll \frac{1}{a_{p}} \ll 1
$$

and the equation (22) for determining $\rho_{0}$ can also be re-expressed as

$$
\frac{\frac{J^{2}}{\rho_{0}^{2}}}{\sqrt{1+\frac{J^{2}}{\rho_{0}^{2}}}}=(7-p) \frac{\lambda}{\rho_{0}^{7-p}} \ll 1,
$$

where we have used the condition (44). This must imply $\frac{J}{\rho_{0}} \ll 1$ and therefore to leading order we have from (45) $\rho_{0} \approx J\left[(7-p) \frac{\lambda}{J^{7-p}}\right]^{\frac{1}{5-p}}$. Combining this with the constraint $\rho_{0} \gg$ $\left(a_{p} \lambda\right)^{1 /(7-p)}$, we have

$$
\frac{J}{\lambda^{\frac{1}{7-p}}} \ll \sqrt{\frac{7-p}{a_{p}^{\frac{5-p}{7-p}}}} \ll 1 .
$$

The maximal value of the potential can now be estimated as

$$
V_{\mathrm{eff}}\left(\rho_{0}\right) \approx 1+\frac{5-p}{2(7-p)^{\frac{7-p}{5-p}}}\left(\frac{J^{7-p}}{\lambda}\right)^{\frac{2}{5-p}}>1
$$

Note that $p=4$ is just a special case here but neither $p=5$ nor $p=6$. So the conditions for having unbound orbits and avoiding the brane annihilation are $V_{\text {eff }}\left(\rho_{0}\right)>H \geq 1$ and (46).

In summary, we have analyzed the dynamical behavior of $\mathrm{Dp} / \overline{\mathrm{D}} \mathrm{p}$ system for $0 \leq p \leq 6$ via its corresponding effective potential in the region of interest stated earlier. We have determined the conditions for which the underlying requirements are met in each case. We find that only for $p=6$ case, there exist both bound orbits and unbound orbits and for all other cases considered only unbound orbits exist. In particular, we find that the dimensionless ratio $J / \lambda^{1 /(7-p)}$ for $p=6, p=5$ and the rest cases considered is very different. Explicitly, $J / \lambda \gg 1$ for $p=6$, $J / \lambda^{1 / 2}$ is bigger than but very close to $\sqrt{2}$ for $p=5$, while $J / \lambda^{1 /(7-p)} \ll 1$ for $0 \leq p \leq 4$. Each of these indicates that the potential well depth $(\mathrm{p}=6)$ or potential barrier height $(0 \leq p \leq 5)$ is very close to its asymptotic value of unit at $\rho \rightarrow \infty$, and therefore the stability of the respective classical orbits needs to be addressed. Given our understanding of the potential behavior and without going into detail, one expects that the classically allowed unbound orbits for $0 \leq p \leq 5$ 
will not be stable quantum mechanically as well as non-perturbatively (for example through tunneling) and even under classical perturbation, and the brane and anti-brane annihilation seems to be inevitable. However, for $p=6$ case, even though the classical bound orbits and those unbound orbits with $H$ near by unit can exchange their role under classical perturbation as well as quantum mechanically, the brane and anti-brane annihilation seems to be remote 8 . In other words, we don't expect to have the brane annihilation in this case in general if the reduced angular momentum $J \gg \lambda$ with $\lambda$ the stringy parameter.

\section{The $\mathrm{D}_{6} / \overline{\mathrm{D}}_{6}$ System}

It is not easy to solve the orbital equation (19) to give explicit and analytical orbits for a general $p(0 \leq p \leq 6)$. However, this can be done with easy for $p=6$. We will solve the orbital equation (19) directly for $p=6$ as an explicit example, analyze the corresponding dynamical behavior of the orbits and find the relevant constraints which validate the region of interest under consideration. We will find that these constraints are in complete agreement with what we found in the previous section based purely on the effective potential. For this, let us begin with (19) for $p=6$ which is

$$
\frac{d \rho}{d \theta}= \pm \frac{\rho^{2}}{J} \sqrt{\left(H+\frac{\lambda}{\rho}\right)^{2}-\left(1+\frac{J^{2}}{\rho^{2}}\right)}
$$

where the \pm correlate to the signs of $\dot{\rho}$ once we choose $J>0$, i.e., $\dot{\theta}>0$. The above equation can be solved to give

$$
\rho=\left\{\begin{array}{cc}
\frac{2 A}{Q \sin \left[ \pm \frac{\sqrt{-A}}{J}\left(\theta-\theta_{0}\right)\right]-B}, & J>\lambda \\
\frac{4 J^{2} B}{B^{2}\left(\theta-\theta_{0}\right)^{2}-4 J^{2} C}, & J=\lambda \\
\frac{4 A J \exp \left[\mp \frac{\sqrt{A}}{J}\left(\theta-\theta_{0}\right)\right]}{\left[J \exp \left[\mp \frac{\sqrt{A}}{J}\left(\theta-\theta_{0}\right)\right]-B\right]^{2}-4 A C}, & J<\lambda
\end{array}\right.
$$

where $\lambda=g_{s} \sqrt{\alpha^{\prime}} / 4=\sqrt{\alpha^{\prime}} / a_{6}$ and

$$
A=\lambda^{2}-J^{2}, \quad B=2 \lambda H, \quad C=H^{2}-1, \quad Q=2 \sqrt{J^{2}\left(H^{2}-1\right)+\lambda^{2}} .
$$

The reduced angular momentum and Hamiltonian are given by (16) and (17) for $p=6$, respectively. For convenience, we collect them here

$$
J=\frac{\rho^{2} \dot{\theta}}{\sqrt{1-\left(\dot{\rho}^{2}+\rho^{2} \dot{\theta}^{2}\right)}}, \quad H=\frac{\sqrt{1+\frac{J^{2}}{\rho^{2}}}}{\sqrt{1-\dot{\rho}^{2}}}-\frac{\lambda}{\rho},
$$

\footnotetext{
${ }^{8}$ Our focus here is on the classical orbits and the other issues of stability such as their possible decays at quantum level discussed in [6] are beyond the scope of the paper. Even so, given what has been considered there, we expect that the quantum stability can still hold in the present case since in our case $g_{s} N \ll 1$.
} 
with $H>0$ in the region of interest.

It is not difficult to examine the solutions (49) that the $\rho$ can be made as small as one wishes for large enough $\theta$ for both $J=\lambda$ case and $J<\lambda$ case. This is also true for either sign in the latter case. Therefore these orbits inevitably lead to the annihilation of brane and anti-brane and are not in the interest of this paper. For this reason, we will then drop them from further consideration and focus on the case of $J>\lambda$ from now on.

So the relevant solution is now

$$
\rho=\frac{-2 A}{B-Q \sin \left[\frac{\sqrt{-A}}{J}\left(\theta-\theta_{0}\right)\right]},
$$

where we for certainty take the ' + ' sign for the $J>\lambda$ case in (49) and the ' - ' sign can be obtained by sending $\theta-\theta_{0} \rightarrow-\left(\theta-\theta_{0}\right)$. Let us analyze the solution in two separate cases.

i) If $B>Q$, we have then the following constraint for $H$ as

$$
\sqrt{1-\frac{\lambda^{2}}{J^{2}}} \leq H<1
$$

where the left side bound is from the non-negative requirement of the quantity in the squareroot of $Q$ in (50) and the right side bound is from $B>Q$ and the fact $H>0$. This is exactly the same constraint as (29) derived in the previous section. It is now obvious from (52) that $\rho$ has both a maximum and a minimum as

$$
\begin{array}{rlrl}
\rho_{\max } & =\frac{-2 A}{B-Q}, & & \text { when } \theta-\theta_{0}=\frac{J}{\sqrt{-A}} \frac{\pi}{2} \\
\rho_{\min }=\frac{-2 A}{B+Q}, & \text { when } \theta-\theta_{0}=\frac{J}{\sqrt{-A}} \frac{3 \pi}{2} .
\end{array}
$$

Note that the orbit is a bound one but it will not be closed in general unless $\frac{J}{\sqrt{-A}}=\frac{m}{n}$ with $m, n$ integers, i.e., with a rational ratio. With (50) and (53), we have from (55)

$$
\rho_{\min }=\frac{J^{2}-\lambda^{2}}{\lambda H+\sqrt{\lambda^{2}-J^{2}\left(1-H^{2}\right)}}>\frac{J^{2}-\lambda^{2}}{2 \lambda} .
$$

To avoid the brane annihilation, we need the minimal brane separation to satisfy $\rho_{\min } \gg$ $\sqrt{\alpha^{\prime}}=a_{6} \lambda$ where in the last equality we have used the expression for $\lambda$ given earlier. This can be automatically satisfied if we have $\frac{J^{2}-\lambda^{2}}{2 \lambda} \gg a_{6} \lambda$, considering the above inequality. The resulting constraint is just (27) derived in the previous section.

ii) If $0<B \leq Q$, we have $H \geq 1$ from the explicit expressions of $B$ and $Q$ given in (50) and from the fact $H>0$. It is obvious from the solution (52) that the orbit is now unbound. We have

$$
\begin{array}{ll}
\rho \rightarrow \infty, & \text { when } \theta-\theta_{0}=\frac{J}{\sqrt{-A}}\left(\pi-\arcsin \frac{B}{Q}\right) \\
\rho_{\min }=\frac{-2 A}{B+Q}, & \text { when } \theta-\theta_{0}=\frac{J}{\sqrt{-A}} \frac{3 \pi}{2} .
\end{array}
$$


To avoid the brane annihilation, we need to have

$$
a_{6} \lambda \ll \rho_{\min }=\frac{J^{2}-\lambda^{2}}{\lambda H+\sqrt{J^{2}\left(H^{2}-1\right)+\lambda^{2}}} \leq \frac{J^{2}-\lambda^{2}}{2 \lambda},
$$

where we have used the explicit expression for $\rho_{\min }$ from (58) and $H \geq 1$ for the second inequality which will give the same constraint between $J$ and $\lambda$ as (27). The constraint on $H$ can be determined from the first inequality above and combining with $H \geq 1$ we have

$$
1 \leq H \ll \sqrt{1+\frac{J^{2}}{\lambda^{2} a_{6}^{2}}}-\frac{1}{a_{6}} .
$$

Once again, we obtain the same constraints on $J$ and $H$ as those derived in the previous section.

\section{Conclusion}

We study the dynamical behavior of a pair of Dp-brane and anti Dp-brane $(0 \leq p \leq 6)$ moving parallel to each other in the region where the brane and anti-brane annihilation will not occur and the low energy description is valid. We find that the classical orbits can indeed exist under conditions specified in the previous sections and are in general unbound except for $p=6$ where bound orbits can exist. However, these unbound orbits except for $p=6$ case are in general unstable quantum mechanically as well as non-perturbatively and even under classical perturbation, and therefore the brane and anti-brane annihilation seems to be inevitable. For $p=6$, we don't expect that the brane and anti-brane annihilate each other even though bound orbits and unbound orbits with $H$ nearby unit can exchange their role under classical perturbation as well as quantum mechanically. The non-linearity of DBI action for D-branes plays an important role in the case of $p=5$ while it has only a quantitative effect for all the other cases under consideration.

\section{Acknowledgements:}

We thank Rong-Jun Wu for reading the manuscript and pointing out a few typos in the original version. We acknowledge support by grants from the Chinese Academy of Sciences, a grant from 973 Program with grant No: 2007CB815401 and grants from the NSF of China with Grant No:10588503 and 10535060.

\section{References}

[1] A. Dabholkar, G. W. Gibbons, J. A. Harvey and F. Ruiz Ruiz, "SUPERSTRINGS AND SOLITONS," Nucl. Phys. B 340, 33 (1990). 
[2] M. J. Duff, R. R. Khuri and J. X. Lu, "String solitons," Phys. Rept. 259, 213 (1995) arXiv:hep-th/9412184.

[3] J. Polchinski, "Dirichlet-Branes and Ramond-Ramond Charges," Phys. Rev. Lett. 75, 4724 (1995) arXiv:hep-th/9510017].

[4] Banks and Susskind, "Brane-Antibrane Forces," arXiv:hep-th/9511194.

[5] J. X. Lu, Bo Ning, Shibaji Roy and Shan-Shan Xu, "On Brane-Antibrane Forces," JHEP 0708, 042 (2007), arXiv:0705.3709 [hep-th].

[6] C. P. Burgess, P. Martineau, F. Quevedo and R. Rabadan, "Branonium," JHEP 0306, 037 (2003) arXiv:hep-th/0303170.

[7] C. P. Burgess, N. E. Grandi, F. Quevedo and R. Rabadan, "D-brane chemistry," JHEP 0401, 067 (2004) arXiv:hep-th/0310010. 